\title{
Integrated Lung Field Segmentation of Injured Region with Anatomical Structure Analysis by Failure-Recovery Algorithm from Chest CT Images
}

\author{
Yuma Iwao $^{\mathrm{a}}$, Toshiyuki Gotoh ${ }^{\mathrm{a}}$, Seiichiro Kagei ${ }^{\mathrm{a}}$, Tae Iwasawa ${ }^{\mathrm{b}}$, \\ Marcos de Sales Guerra Tsuzuki ${ }^{\mathrm{c}}$ \\ ${ }^{a}$ Yokohama National University, 79-1 Tokiwadai, Hodogaya-ku, Yokohama-shi, \\ Kanagawa, 240-8501 Japan \\ ${ }^{b}$ Kanagawa Cardiovascular Respiratory Center, Kanazawa-ku, Yokohama-shi, \\ Kanagawa, 236-0051 Japan \\ ${ }^{c}$ Escola Politécnica da Universidade de São Paulo, Brazil. \\ Mechatronics and Mechanical Systems Engineering Department \\ Computational Geometry Laboratory
}

\begin{abstract}
This work proposes a functionality for computerized tomography (CT) based investigation of diffuse lung diseases diagnosis that enables the evaluation of the disease from lung anatomical structures. Automated methods for segmenting several anatomy structures in chest CT are proposed: namely the lobe lungs, airway tree and pulmonary vessel tree. The airway and pulmonary vessel trees are segmented using a failure tracking and recovery algorithm. The algorithm checks intermediary results consistence, backtrack to a history position if a failure is detected. The quality of the result is improved while reducing the processing time even for subjects with lung diseases. The pulmonary vessels are segmented through the same algorithm with different seed points. The seed for the airway tree segmentation is within the tracheal tube, and the seed for the pulmonary vessels segmentation is within the heart. The algorithm is tested with CT images acquired from four distinct types of subjects: healthy, idiopathic interstitial pneumonia (IIPs), usual interstitial pneumonia (UIP) and chronic obstructive pulmonary disease (COPD). The main bronchi are found in the segmented airway and the associated lung lobes are determined. Combining the segmented lung lobes and the diff lung diseases classification, it is possible to quantify how much and where each lobe is injured. The results were compared with a conventional $3 \mathrm{D}$ region
\end{abstract}


growing algorithm and commercial systems. Several results were compared to medical doctor evaluations: inter-lobe fissure, percentage of lung lobe that is injured and lung and lobe volumes. The algorithm proposed was evaluated to be robust enough to segment the cases studied.

Keywords: Airway segmentation, pulmonary vessels segmentation, diffuse lung diseases classification.

\section{Introduction}

Segmentation of the pulmonary lobes is important to locate parenchymal disease inside the lungs and to quantify its distribution. The segmentation issue becomes even more challenging with regard to the high variability of the computerized tomography (CT) image in clinical practice due to the use of different CT scanners and protocols. One might think that the first step for lung structure recognition is to extract inter-lobe fissures and use them to divide the lung regions. However, the inter-lobe fissures are very thin surfaces with light density in CT images and difficult to be observed from original CT images even by human experts [1].

The left lung is divided into two lobes, upper and lower, by an inter-lobe fissure. The right lung is divided into three lobes, upper, middle and lower, by two inter-lobe fissures. Pulmonary fissures are often only incomplete or not clearly visible in the CT image because of severe lung diseases and low image resolution. Previous approaches to lobe segmentation can be roughly divided into two groups: direct and indirect. The former approaches consist of methods that search for the fissures based on gray-level information present in the image $[2,3,4,5]$, while the latter approaches consist of methods that use information from other anatomical structures to approximate the location of the fissures $[6,7]$. Different techniques were used by the direct methods; Zhang et al. [4] presented a method for automatic segmentation of the oblique fissures using an atlas-based initialization procedure, in which the anatomic atlas is created by manually delineating the fissures on a number of subjects. Wiemker et al. [2] proposed an automatic segmentation approach based on 3D filtering of the image data. More recently, van Rikxoort et al. [5] described a nearest-neighbor classifier approach to identify voxels on the fissures.

Among the indirect approaches, Kuhnigk et al. [6] proposed a method for the indirect estimation of the lobes, using information from the segmented vasculature. In a similar approach, Zhou et al. [7] reported a method based 
on the Voronoi division of the lungs using the lobar bronchi. Neither indirect approach was evaluated by their authors. The approach proposed herein is classified as indirect. As a secondary objective, we also propose a 3D segmentation algorithm that can extract the airways and lung vessels. Such lung anatomical structures are used to determine the fissure location.

3D segmentation of the airway from multi-slice CT is useful for analyzing airway compression and obstruction caused by pathology. The expected benefits are related to diagnosis improvement of airway pathologies, preoperative planning and follow-up. Accurate lung segmentation allows the detection and quantification of abnormalities within the lungs. It is a challenging problem due to the inhomogeneity of the bronchial lumen and bronchial wall grey levels along different subdivision orders. Several methods have been presented in the literature for this task. The methods proposed can be classified into three main categories: 1) 2D/3D techniques detect potential airway sections on 2D axial images and then perform the selection of the candidates which will provide the 3D reconstruction [8]. 2) 3D region growing techniques act directly on the 3D image. They combine thresholding and 3D propagation procedures in order to generate an airway tree reconstructed set, connected to a seed defined inside the trachea. With these techniques, the reconstruction accuracy depends directly on the threshold setting. Adaptive thresholding cannot ensure a reliable reconstruction due to the inhomogeneity of the airway lumen along the tree structure [9]. 3) hybrid methods combine 3D region growing and 2D segmentation approaches to detect more airway candidates and to obtain smoother edges. Even if they provide better results [10], the main limitation of these techniques remains their inability to reconstruct high order bronchi: the 2D and 3D segmentation phases being independent, their common limitations are inherited by the hybrid method.

Extraction of vessels in CT is a key component for the diagnosis of vascular diseases, such as stenosis, hypertension and embolism. The vessel tree is also an important cue for registering images of the same patient. Another role of vessel tree extraction is an aid to improve detection of other structures, such as lung nodules, which has shown to help reduce false positives [11, 12]. Different approaches have been used to segment lung vessels. Kirbas and Quek [12] divided vessel segmentation algorithms and techniques into six main categories: (1) pattern recognition techniques, (2) model-based approaches, (3) tracking-based approaches, (4) artificial intelligence-based approaches, (5) neural network-based approaches, and (6) miscellaneous tubelike object detection approaches. 
Although the correct diagnosis of diffuse lung disease is very important, it is one of the most difficult tasks for radiologists, because the contrast of lesions is often low and the patterns of opacities are very complex. Diffuse lung disease represents a large and heterogeneous group of disorders. In the management of patients with diffuse lung diseases, it is important to define the extent of disease present, particularly if the patient is being monitored for disease progression or response to treatment. Since lung diseases are commonly nonuniform in their distribution, physiologic measurements may underestimate the extent of lung diseases. The method chosen for feature extraction is clearly critical to the success of texture classification. In this work, diffuse lung diseases are classified using the algorithm proposed by Asakura et al. [13], which was already tested by several hospitals with different CT scanners and protocols [14].

This work investigates an anatomical classification technique for lung structures and integrates it with the diffuse lung disease classification approach proposed by Asakura et al. [13], such system was already tested by several hospitals with different CT scanners and protocols [14]. This work is structured as follows. Section 2 explains the improved algorithm to classify diffuse lung diseases. Section 3 briefly explains the system proposed to integrate the determined lung structures with classified diffuse lung diseases. The algorithm proposed to segment airway, pulmonary vessel and lungs is also explained. Section 4 presents some results; the conclusions and future works are in section 5 .

\section{Classification of Diffuse Lung Diseases}

Texture classification has been a significant research topic in image processing, particularly in medical image analysis, and many features have been proposed to represent texture. Five major categories of features for texture identification have been proposed: statistical, geometrical, structural, model based and signal processing [15]. In order to classify diffuse lung diseases the algorithm proposed by [13] was improved such that images from multiple CT scanners can be processed using the same database. Fig. 1 shows the flow of the improved approach.

Initially, a calibration process is executed for all images to normalize the standard deviation (see Fig. 1). Every pixel from the CT images is linearly interpolated between the values obtained from water and air phantoms. The CT images from subjects and phantoms are obtained on the same day. The 


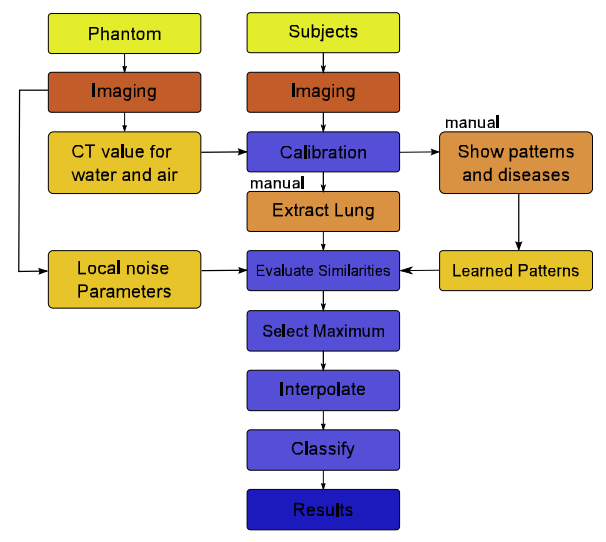

Figure 1: Flow of the improved classifier.

user defines a set of patterns associated to each diffuse lung disease, called learned patterns. Similarities between the learned patterns and regions in the CT image are evaluated using a Gaussian histogram normalized correlation method and a local noise model of the chest CT images. Since the fluctuation of the CT distributes in a shape similar to a Gaussian distribution [16], the local noise of a CT pixel is assumed to be spatially random with the Gaussian distribution.

After the application of the Gaussian convolution filter, the normalized correlations between the estimated distribution of $\mathrm{CT}$ regions and those of learning patterns with the diseased classes are calculated by the following equation

$$
r(\boldsymbol{\alpha}, \boldsymbol{\beta})=\frac{1}{A(\boldsymbol{\alpha}) A(\boldsymbol{\beta})} \sum_{\alpha \in \boldsymbol{\alpha}} \sum_{\beta \in \boldsymbol{\beta}} l(\alpha, \beta)
$$

where

$$
l(\alpha, \beta)=\frac{1}{2 \sqrt{\pi} \sigma} \exp \left\{-\left(\frac{(\alpha-\beta)^{2}}{4 \sigma^{2}}\right)\right\}
$$

where $A(\boldsymbol{\alpha})$ and $A(\boldsymbol{\beta})$ are the histograms of the learning pattern $\boldsymbol{\alpha}$ and the $\mathrm{CT}$ region $\boldsymbol{\beta}$, and $\sigma$ is the noise model standard deviation. In this paper, $\sigma=23.35 \mathrm{HU}$ (Hounsfield Units) is used. This expression allows the comparison of regions in the CT images with the learned patterns.

Furthermore, because of gravity the blood flow can undergo variations that can globally influence the CT image. To reduce such influence, the user 


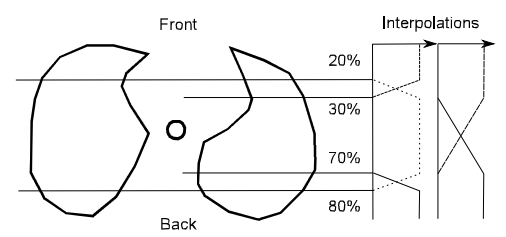

(a)

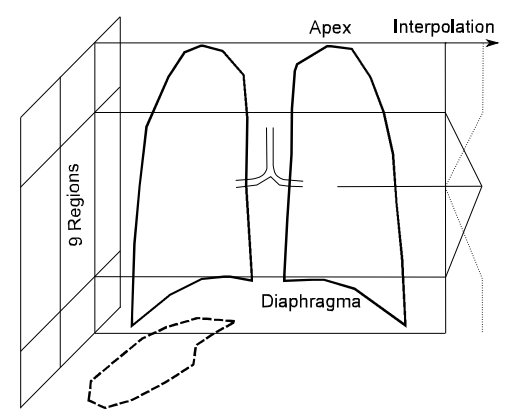

(b)

Figure 2: Nine regions where templates are defined to reduce blood flow gravity influence.

divides the lung into nine regions and defines learned patterns for each one of these nine regions (see Fig. 2). Finally, if the learned patterns are directly used; they might present some discontinuities. To overcome this possibility, depending on the region of the CT image analyzed, an interpolation is executed.

The classifier has been used in different applications: evaluating the utility CT imaging to understand the response to pirfenidone therapy [17], the use of CT images to evaluate the relation between the severity of idiopathic pulmonary fibrosis (IPF) and the incidence of pneumothorax [18], among others.

\section{Proposed System}

The proposed system architecture is shown in Fig. 3. The system proposed segments the lung 3D CT image into lung boundary, airway tree and lung vessel tree. The airway tree is anatomically classified into 5 lobes. The lung vessel tree is also classified using the airway tree classification. For each voxel in the lung vessel tree, the nearest airway tree voxel is determined and 


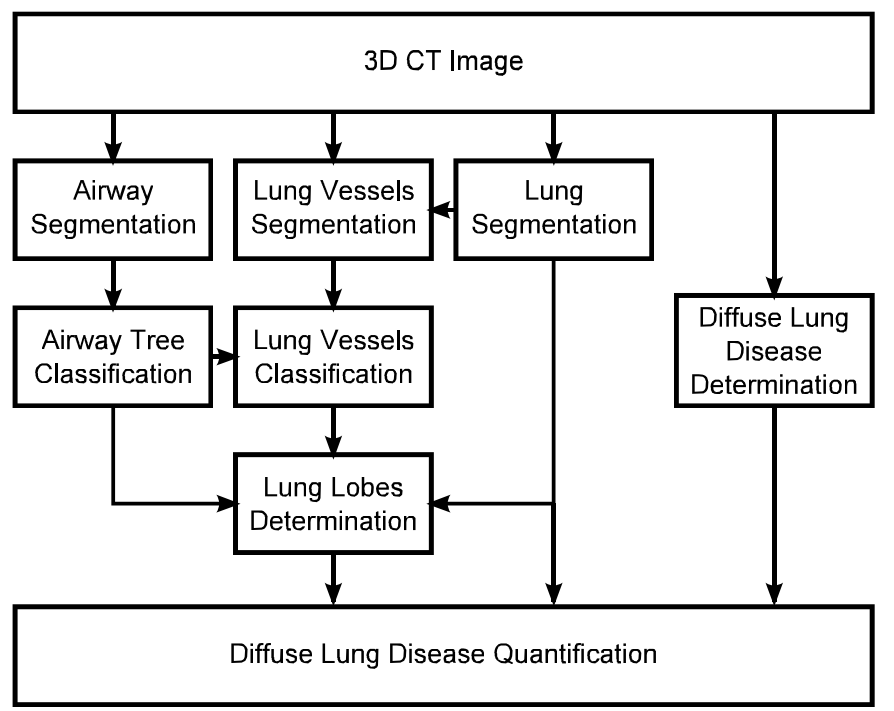

Figure 3: Proposed system architecture.

both voxels are set to have the same lobe classification. A 3D Voronoi is used to determine which part of the lung corresponds to each lung lobe.

\subsection{Airway Segmentation by Failure Tracking and Recovery Algorithm}

3D region growing algorithms, taking advantage of speed and simplicity, is widely used as a method for airway segmentation from CT images [19]. The algorithm is based on the fact that the bronchial lumen has low intensity and it is surrounded by the bronchial wall whose intensity is relatively high in a CT image. However, the thickness of a bronchial wall becomes thinner in the peripheral area of the lung and its intensity becomes lower (see Fig. 4). The partial volume effect creates holes in bronchial walls in CT images. Such regions where the boundary is not clear cannot be segmented correctly by a region growing method. Several methods have been proposed to overcome this problem: region growing method with adaptive threshold [8], rule based detection [10] and others [20]. Consequently, these methods proposed avoid failures by increasing complexity and computational cost.

In this work, a failure tracking and recovery algorithm that admits failures is proposed. The algorithm consists of a recovering process, history storage (with plane and branch data stacking) and the main process. It is possible to backtrack to a previous state in history and re-start the processing from a safe 


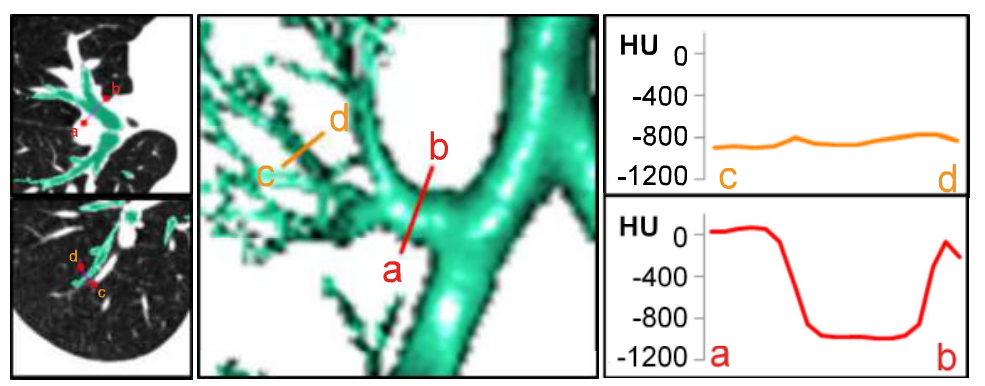

Figure 4: Most existing airway segmentation methods are based on region growing, with the assumption that the airway has low intensity and is surrounded by higher intensity airway walls (section $a-b$ ). The contrast between the airway and its surroundings is sometimes very low, due to noise or pathologies (section $c$ - $d$ ).

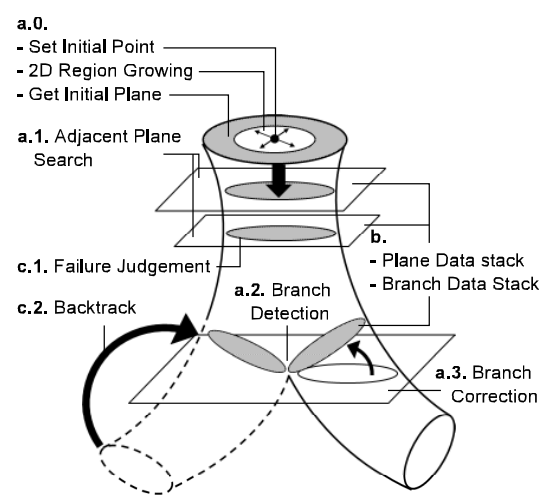

(a)

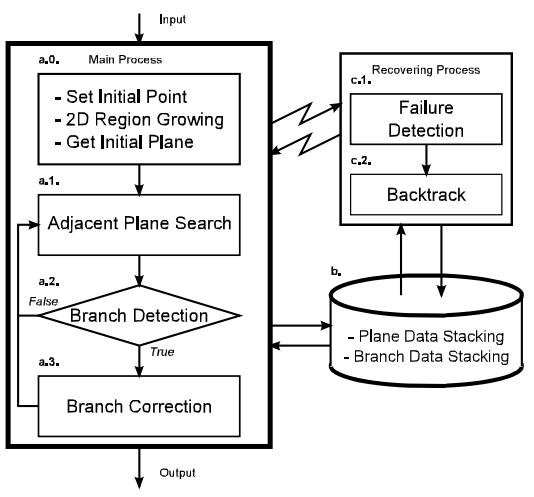

(b)

Figure 5: Failure Tracking and Recovery model for airway extraction. (a) Algorithm phases and their associated information. (b) Flowchart of the algorithm proposed. 


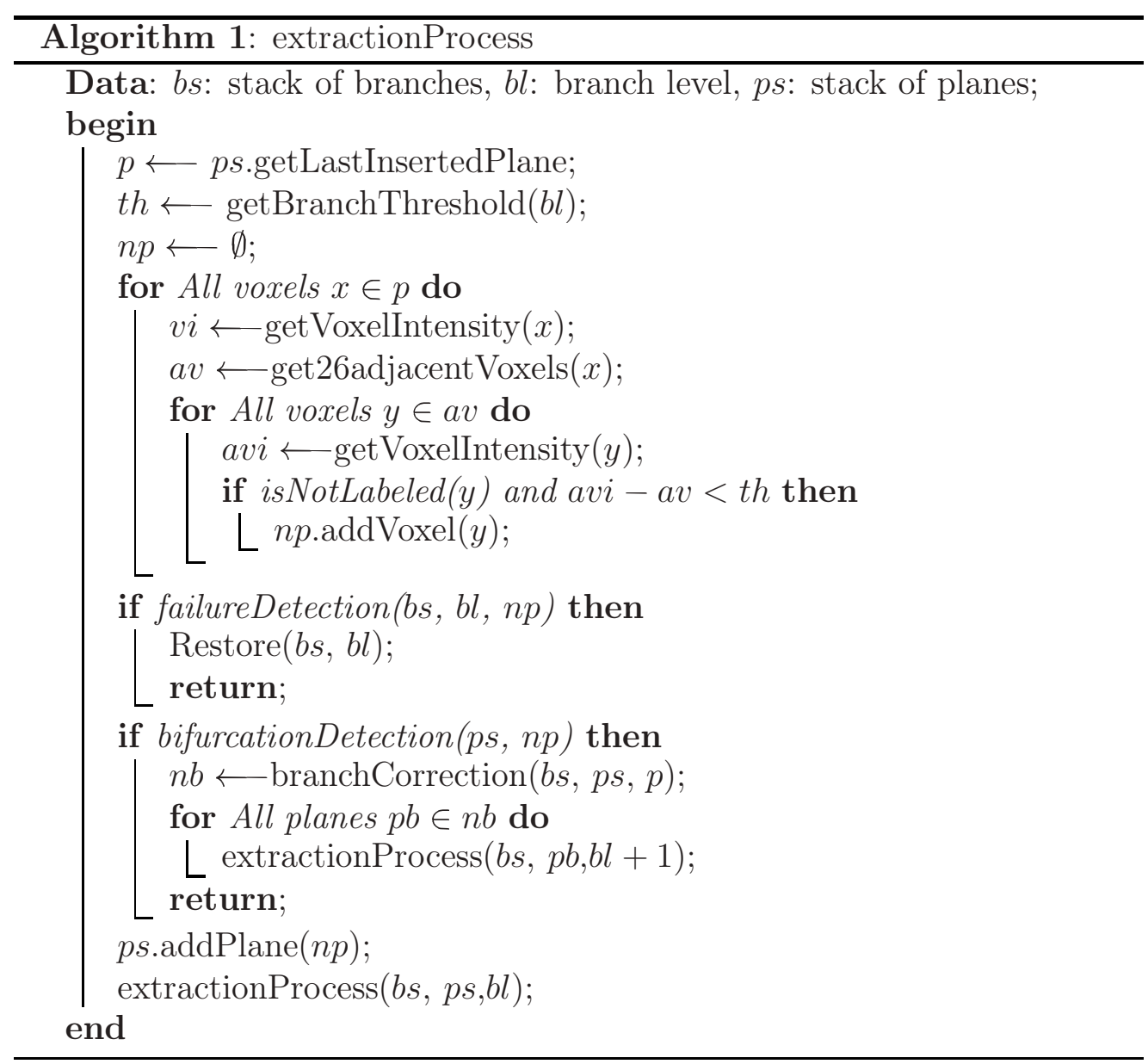




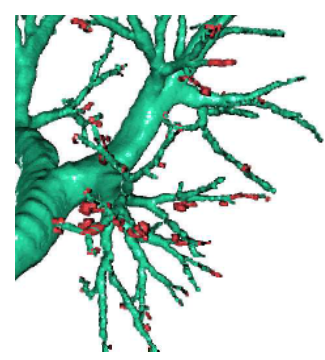

(a)

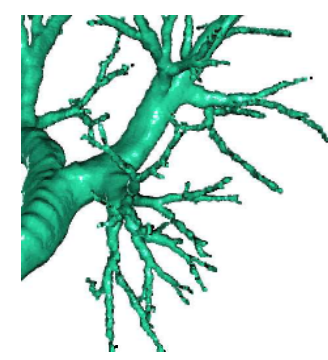

(b)

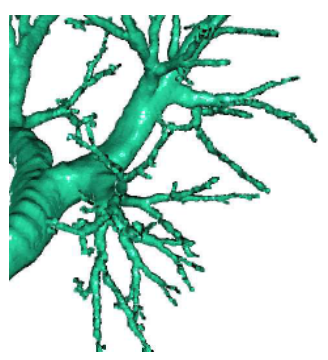

(c)

Figure 6: (a) Airway tree with some failures detected in red. (b) Airway tree with all detected failures backtracked, after recovering phase. (c) The final situation of the airway tree after reprocessing.

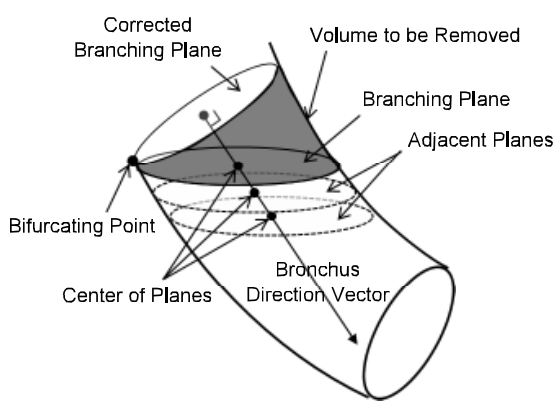

(a)

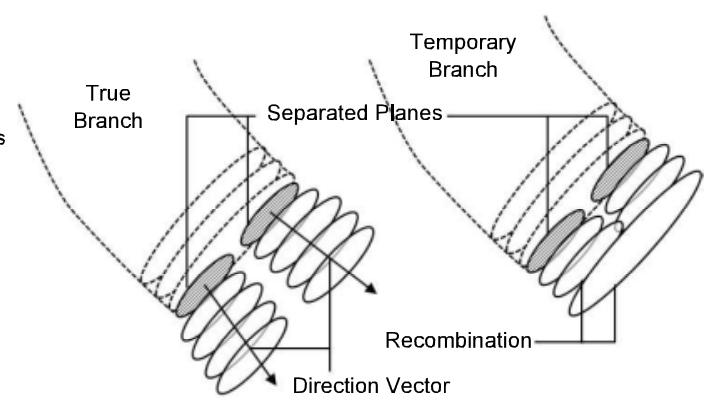

(b)

Figure 7: (a) Elements associated with a branch. (b) Possible recombination of two branches. 
state when a failure is detected. Its flowchart is shown in Fig. 5.(b). Initially, a seed within the tracheal lumen is selected by the user. A threshold with a large enough value is fixed. Starting from the current position adjacent pixels in the current horizontal plane are analyzed (see Fig. 5.(a)). A first horizontal plane is defined. Adjacent planes are searched recursively (see Algorithm. 1) and stored in a stack.

Validation is the crucial point of the algorithm because it is responsible for rejecting the current algorithm state and forcing the algorithm to return to a previous state with new threshold value (the threshold value is decremented). In the current implementation, the validation procedure uses two criteria: volume and radius. Generally, radius and volume do not change rapidly. When a new plane is determined, the algorithm checks if the new plane and the stacked planes are a viable branch. Fig. 6.(a) shows several detected failures in red. If it is not a viable branch, the threshold value for the current branch is decremented and the stack of planes is reset and just the first plane remains (see Fig. 6.(b)). If the threshold value becomes null then the current branch is removed from the data structure. The algorithm starts again with a new decremented threshold value. Fig. 6.(c) shows the final result.

A branch is represented by a stack of planes in which planes are recursively pushed considering the direction defined by the firstly included plane. Even disconnected planes can be pushed into the stack. Disconnected planes can be recombined if a bifurcation is not detected (see Fig. 7.(b)). If a bifurcation is detected, the stack of planes is divided into three parts: one root and two child branches. The current branch is finished and stored in the branch stack. The initial planes for the new bifurcating branches are determined. Fig. 7.(a) explains the procedure. The very first planes are horizontal and the following initial planes associated with new branches can have any angle.

\subsection{Lung Segmentation}

The identification of the lungs in thoracic CT images is a prerequisite for almost any kind of CT-based computer assistance in pulmonary radiology. In particular, the segmentation of the left and right lung is the first step towards a region based quantification of lung parenchyma. Due to the high density difference between the air-filled lung regions and soft tissue in CT images, the pulmonary airspace can usually be identified without greater efforts. For use in a clinical environment, lung segmentation must furthermore be robust against pathological alterations, have acceptable time and memory requirements [21]. Here, the lung is segmented using a conventional 3D 


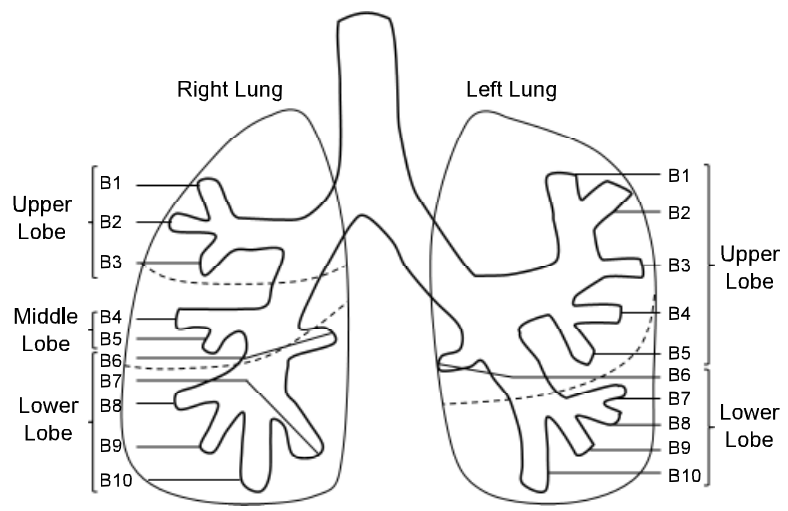

Figure 8: Lung structural elements.

region algorithm.

\subsection{Lung Vessel Extraction}

In this work, the same algorithm used to segment the airway tree is applied to lung vessels segmentation, due to the high contrast between blood vessels and lung parenchyma. The algorithm for lung vessels segmentation has few differences: the vessels can have several ramifications and the validation rules are not the same. This way, at least two starting points are used and at least two vessel trees are determined. After the processing, the vessel trees are combined. The same validation rules considering volume and radius are used, and additional pulmonary vessels must be internal to the lung boundary without intersecting the airway tree.

\subsection{Lung Region Structural Classification}

The airway tree structure is automatically classified into five lobes herein. The classification is based on the directions of the initial bronchus. Fig. 8 [22] shows an anatomical atlas with approximate bronchus direction for several initial bronchi (until third bifurcation level). The anatomical atlas bronchus directions are used to classify the airway tree determined by the proposed failure tracking and recovery algorithm. The airway tree classification is used to help the lung vessel tree classification. All lung vessel tree voxels are processed and the nearest airway tree voxel is determined, and, temporarily, both voxels are associated with the same classification. Finally, the lung vessel tree is hierarchically tracked, in which the entire subtree is coherently 
Table 1: Characterization of the CT images used in the experiments taken from six subjects. All sequences have $512 \times 512$ pixels. NS $=$ Number of Slices, $\mathbf{S T}=$ Slice Thichness $(\mathrm{mm})$

\begin{tabular}{|l|c|c|}
\hline Name & NS & ST \\
\hline NJ (Normal) & 341 & 1.0 \\
\hline SK (UIP) & 711 & 0.5 \\
\hline KS (COPD) & 346 & 1.0 \\
\hline TK (IIPs) & 681 & 0.5 \\
\hline SY (IIPs) & 541 & 0.5 \\
\hline MJ (COPD) & 346 & 1.0 \\
\hline
\end{tabular}

classified. A subtree must belong exclusively to one lung lobe. A Voronoi diagram is constructed using the airway and vessel trees voxels [23]. The Voronoi regions are classified into lobes according to their original voxel classification. This algorithm is possible because the lobes represent functional units and have their own bronchial and vascular systems.

\section{Results and Validation}

The CT images were obtained from six subjects lying supine: one healthy subject, two patients with idiopathic interstitial pneumonias (IIPs), two patients with chronic obstructive pulmonary disease (COPD) and one patient with usual interstitial pneumonia (UIP) ${ }^{1}$. Table 1 shows several details about the CT images used. The results were validated by comparison against a conventional 3D region growing algorithm, two commercial systems and a medical expert evaluation.

\subsection{Results}

Figures 9.(a)-(f) show the lung extracted by the region growing method. The external surface of some injured lungs might be irregular as a consequence of the destruction of the lung parenchyma (see Figs. 9.(b)-(f)).

\footnotetext{
${ }^{1}$ The protocol was approved by the hospital medical ethics committee of Kanagawa Cardiovascular and Respiratory Center, and informed consent was obtained from each patient.
} 


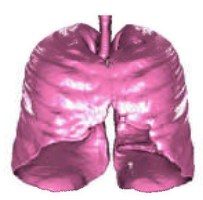

(a)

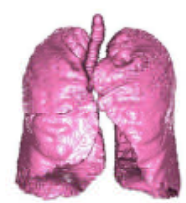

(b)

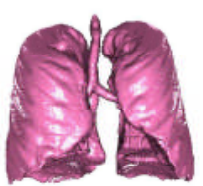

(c)

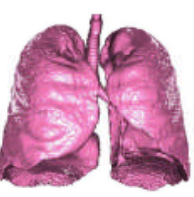

(d)

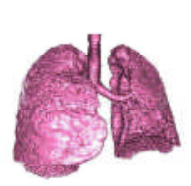

(e)

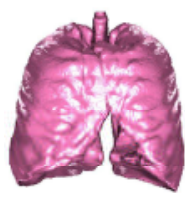

(f)

Figure 9: Extracted lungs. (a) NJ (Normal). (b) SK (UIP). (c) KS (COPD). (d) TK (IIPs). (e) SY (IIPs). (f) MJ (COPD).

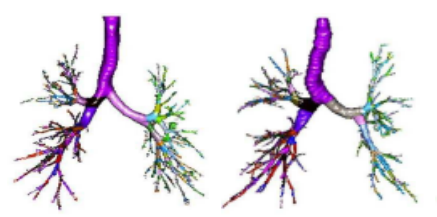

(a)

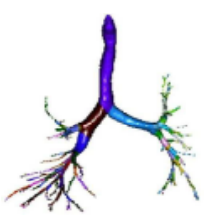

(c)

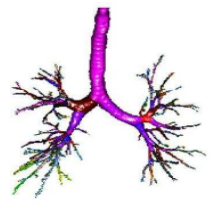

(d)

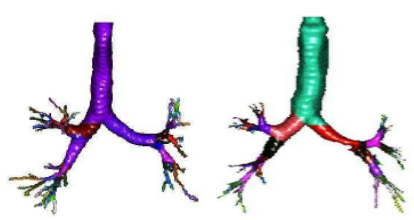

(e)

(f)

Figure 10: Extracted airway trees using the proposed failure tracking and recovery algorithm. (a) NJ (Normal). (b) SK (UIP). (c) KS (COPD). (d) TK (IIPs). (e) SY (IIPs). (f) $\mathrm{MJ}(\mathrm{COPD})$.

Table 2: Airway extraction statistics for the results shown in Fig. 10. AV = airway volume $(c c), \mathbf{A L}=$ airway length $(\mathrm{mm}), \mathbf{N B}=$ number of bronchus, $\mathbf{P T}=$ processing time $(s)$

\begin{tabular}{|c|c|c|c|c|}
\hline Name & AV & AL & NB & PT \\
\hline NJ & 62 & 3,590 & 730 & 0.53 \\
\hline SK & 70 & 3,784 & 741 & 0.79 \\
\hline KS & 53 & 2,103 & 291 & 0.29 \\
\hline TK & 67 & 3,985 & 641 & 0.64 \\
\hline SY & 47 & 1,038 & 217 & 1.09 \\
\hline MJ & 60 & 994 & 163 & 0.25 \\
\hline
\end{tabular}




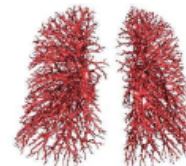

(a)

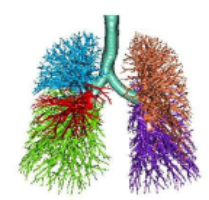

(g)

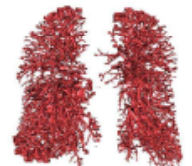

(b)

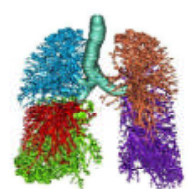

(h)

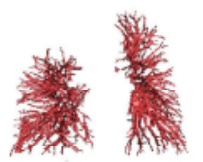

(c)

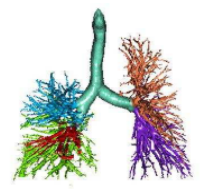

(i)

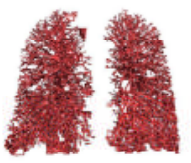

(d)

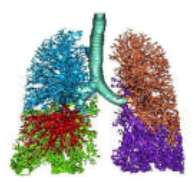

(j)

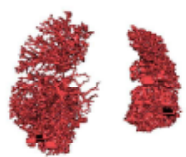

(e)

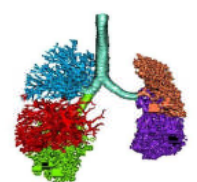

(k)

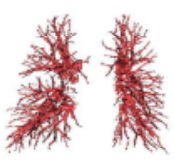

(f)

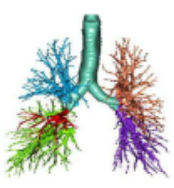

(1)

Figure 11: Extracted vessels and their classification according to lobe regions. (a)-(g) NJ (Normal). (b)-(h) SK (UIP). (c)-(i) KS (COPD). (d)-(j) TK (IIPs). (e)-(k) SY (IIPs). (f)-(l) MJ (COPD).

The result of the proposed failure tracking and recovery algorithm is shown in Figs. 10.(a)-(f). Patients with IIPs might have an atrophy of the lung parenchyma and consequently an increase in the internal region with air. The method proposed showed to be robust to patients in such a condition (see Figs. 10.(d)-(e)). The COPD patient KS has no airway in the right lung upper part (see Fig. 10.(c)). The volume, length and number of branches of the detected airway tree are often used as criteria for assessing the performance of airway tree segmentation algorithms. Table 2 shows the three criteria and their respective processing runtime in seconds.

Figures 11.(a)-(f) show extracted lung vessels. Similar to the airway tree, Table 3 shows some statistics related to lung vessel extraction. Comparing with the bronchus segmentation statistics, the lung vessel tree has a larger volume, longer length and higher processing time. The COPD patient KS has just few vessels in the right lung upper part (see Fig. 11.(c)-(i)). The segmented vessels were classified according to the lung lobes and the result rendered is shown in Figs. 11.(g)-(l). The meaning of the colors are: right upper lobe (blue), right middle lobe (red), right lower lobe (green), left upper lobe (orange) and left lower lobe (purple).

Figures 12.(a)-(f) show the final lobe classification using the same color scheme as for Figs. 11.(g)-(l). Very few segmented bronchus and vessels 
Table 3: Lung vessel extraction statistics (see Fig. 11). VV = lung vessels volume $(c c)$, $\mathbf{N B}=$ number of branches, $\mathbf{V L}=$ lung vessels length $(\mathrm{mm})$ and $\mathbf{P T}=$ processing time $(s)$

\begin{tabular}{|c|c|c|c|r|}
\hline Name & VV & NB & VL & PT \\
\hline NJ & 140 & 1,557 & 13,308 & 1.0 \\
\hline SK & 230 & 4,311 & 17,647 & 11.1 \\
\hline KS & 169 & 2,112 & 13,491 & 2.3 \\
\hline TK & 305 & 5,618 & 21,169 & 17.3 \\
\hline SY & 308 & 6,184 & 9,360 & 6.1 \\
\hline MJ & 120 & 1,565 & 11,149 & 1.1 \\
\hline
\end{tabular}

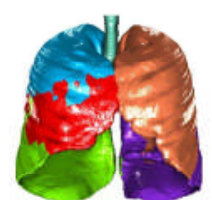

(a)

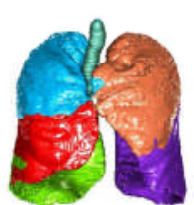

(b)

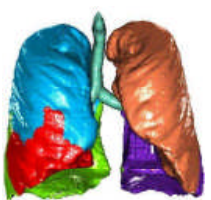

(c)

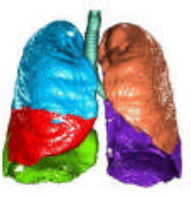

(d)

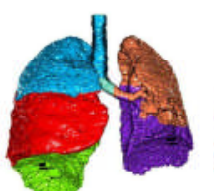

(e)

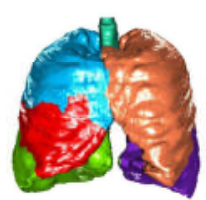

(f)

Figure 12: Lungs classified according to lobe regions. (a) NJ (Normal). (b) SK (UIP). (c) KS (COPD). (d) TK (IIPs). (e) SY (IIPs). (f) MJ (COPD).

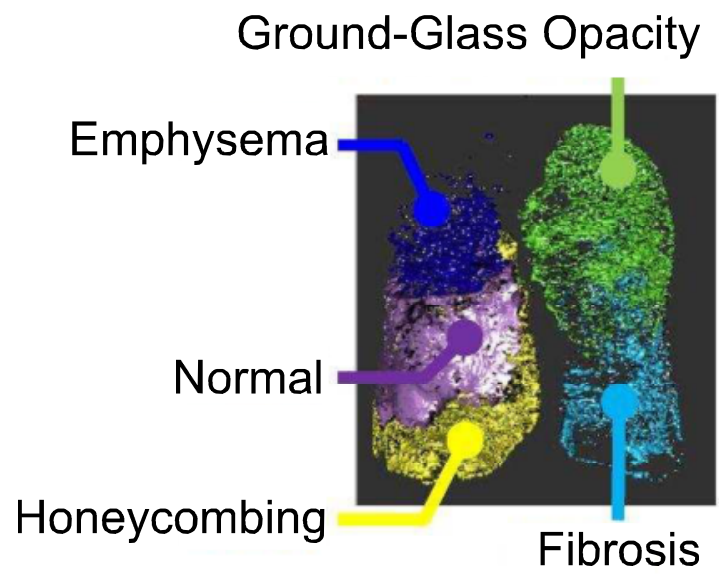

Figure 13: Color scheme used to displa lassified patterns. The regions of ground glass opacity, emphysema, normal, honeycombing and fibrosis are presented on the detected lobes shown in Fig. 14. 


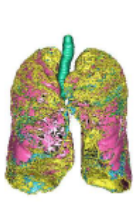

(a)

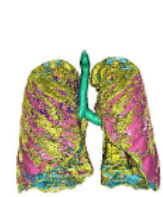

(e)

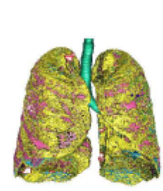

(i)

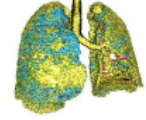

$(\mathrm{m})$

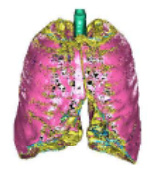

(q)

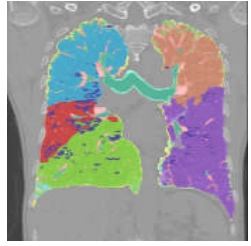

(b)

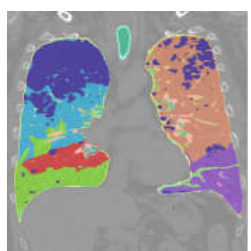

(f)

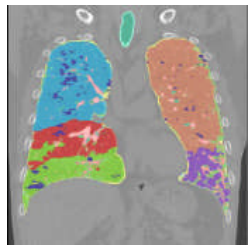

(j)

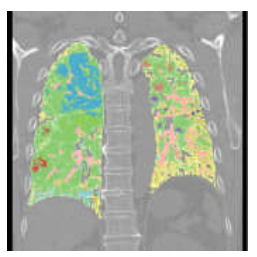

(n)

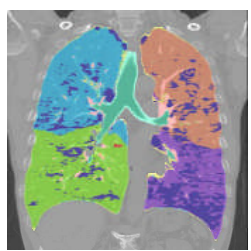

(r)

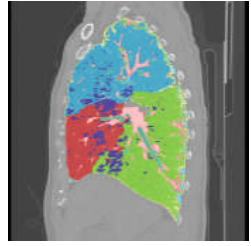

(c)

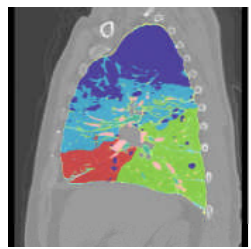

(g)

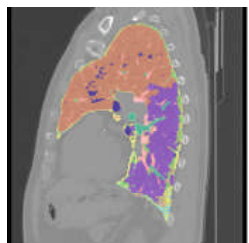

(k)

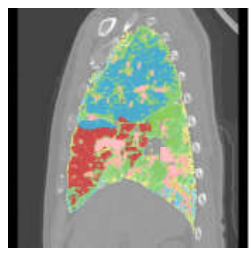

(o)

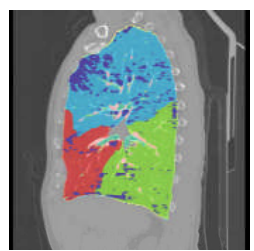

(s)

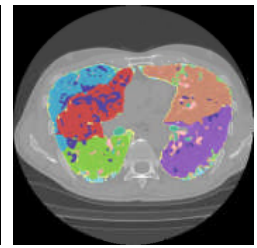

(d)

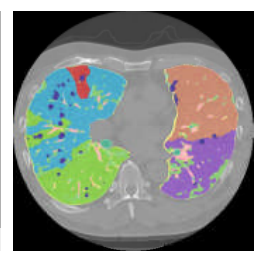

(h)

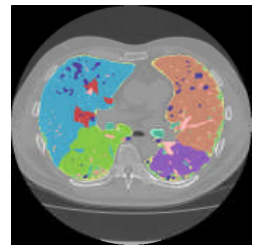

(1)

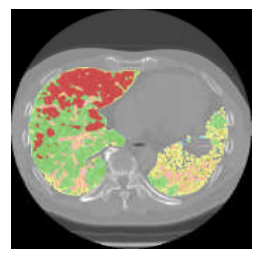

(p)

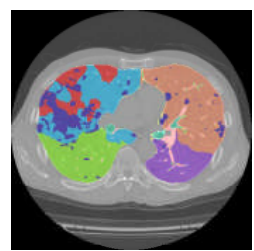

(t)

Figure 14: 3D, coronal, sagittal and axial images with detected diffuse lung disease patterns. (a)-(d) SK (UIP). (e)-(h) KS (COPD). (i)-(l) TK (IIPs). (m)-(p) SY (IIPs). (q)(t) MJ (COPD). The figures show honeycombing and fibrosis separately. For evaluation, honeycombing and fibrosis were considered fibrosis. 
reached near the pleura and, consequently, the region classification near the lung boundary does not seem natural. But it can give a rough idea of where the lung lobes are located.

The classification algorithm proposed by Asakura et al. [13] for diffuse lung diseases was used and the following patterns were classified: normal, ground-glass opacity, emphysema, honeycombing and fibrosis. The color scheme shown in Fig. 13 was adopted. Fig. 14 shows the classification for patients SK (UIP), KS (COPD), TK (IIPs), SY (IIPs) and MJ (COPD). Honeycombing and fibrosis were combined here and considered fibrosis. By combining the lobe classification and the determined diffuse lung disease patterns, it is possible to quantify the percentage of each lobe that is damaged. Table 4 shows a summary of the statistics for the results shown in Fig. 14 . It was possible to verify that the right upper lobe from the KS patient was approximately $60 \%$ injured, and this can be the reason why few bronchus and vessels were extracted in that region.

\subsection{Validation}

The results were validated by five different strategies. 1) A conventional $3 \mathrm{D}$ region growing algorithm to segment the airway tree was implemented and its performance was evaluated and compared with the proposed failure tracking and recovery algorithm. 2) Two type of commercial software were used to segment the airway trees and the results of the fastest system were compared with the ones obtained by the algorithm proposed. 3) The interlobe fissures manually determined by a medical expert and the results were compared with the ones obtained by the proposed system. 4) The commercial software AW 4.6 has a function where it is possible to manually determine the inter-lobe fissure. Based on such manual determination, the AW 4.6 determines the lung lobes volumes. Such volumes are compared with the ones determined by the system proposed. 5) A medical expert analyzed three slices from each of the processed 3D CT images and the amount of damage in the right lower lobe was determined. The same slices were processed by the proposed system and the results were compared.

\subsubsection{Conventional 3D Region Growing Comparison}

The results from processing the conventional 3D region growing algorithm are shown in Figs. 15.(a)-(f). The different colors indicate the bifurcation level. Table 5 shows the segmentation performance of the conventional 3D region growing algorithm. By comparing Tables 2 and 5, it is possible to 
Table 4: Diffuse lung disease patterns percentages for the result shown in Fig. 14. DLDP diffuse lung disease pattern, $\mathbf{L V}=$ Lung Volume, $\mathbf{R U L}=$ right upper lobe, $\mathbf{R M L}=$ right middle lobe, $\mathbf{R L L}=$ right lower lobe, $\mathbf{L U L}=$ left upper lobe, $\mathbf{L L L}=$ left lower lobe, $\mathbf{N o}=$ normal, GGO = ground-glass opacity, Emp $=$ emphysema and $\mathbf{F i b}=$ fibrosis

\begin{tabular}{|c|c|c|c|c|c|c|c|}
\hline Name & DLDP & LV & RUL & RML & RLL & LUL & LLL \\
\hline \multirow{5}{*}{ SK } & No & $85.3 \%$ & $86.4 \%$ & $85.0 \%$ & $82.6 \%$ & $86.5 \%$ & $86.0 \%$ \\
& Emp & $4.3 \%$ & $5.2 \%$ & $11.7 \%$ & $3.5 \%$ & $1.9 \%$ & $2.5 \%$ \\
& GGO & $3.5 \%$ & $2.8 \%$ & $0.9 \%$ & $4.9 \%$ & $4.5 \%$ & $3.4 \%$ \\
& Fib & $6.9 \%$ & $5.6 \%$ & $2.4 \%$ & $9.0 \%$ & $7.1 \%$ & $8.1 \%$ \\
\hline \multirow{5}{*}{ KS } & No & $71.9 \%$ & $45.1 \%$ & $87.5 \%$ & $86.0 \%$ & $86.4 \%$ & $82.8 \%$ \\
& Emp & $18.2 \%$ & $46.9 \%$ & $5.9 \%$ & $6.8 \%$ & $1.4 \%$ & $0.7 \%$ \\
& GGO & $5.0 \%$ & $3.8 \%$ & $3.3 \%$ & $3.3 \%$ & $6.9 \%$ & $6.7 \%$ \\
& Fib & $4.9 \%$ & $4.2 \%$ & $3.3 \%$ & $3.9 \%$ & $5.3 \%$ & $9.8 \%$ \\
\hline \multirow{5}{*}{ TK } & No & $83.6 \%$ & $87.1 \%$ & $90.2 \%$ & $78.1 \%$ & $88.3 \%$ & $71.8 \%$ \\
& Emp & $3.8 \%$ & $6.8 \%$ & $5.4 \%$ & $3.2 \%$ & $2.4 \%$ & $0.5 \%$ \\
& GGO & $6.1 \%$ & $2.7 \%$ & $1.3 \%$ & $9.3 \%$ & $4.6 \%$ & $13.8 \%$ \\
& Fib & $6.5 \%$ & $3.4 \%$ & $3.1 \%$ & $9.4 \%$ & $4.7 \%$ & $13.9 \%$ \\
\hline \multirow{5}{*}{ SY } & No & $31.4 \%$ & $45.8 \%$ & $53.7 \%$ & $6.5 \%$ & $8.4 \%$ & $1.3 \%$ \\
& Emp & $0.1 \%$ & $0.0 \%$ & $0.0 \%$ & $0.1 \%$ & $0.3 \%$ & $0.1 \%$ \\
& GGO & $40.1 \%$ & $38.6 \%$ & $33.2 \%$ & $57.0 \%$ & $42.4 \%$ & $37.0 \%$ \\
& Fib & $28.4 \%$ & $15.6 \%$ & $13.1 \%$ & $36.4 \%$ & $48.9 \%$ & $61.6 \%$ \\
\hline \multirow{5}{*}{ MJ } & No & $83.3 \%$ & $81.1 \%$ & $88.9 \%$ & $89.2 \%$ & $84.9 \%$ & $74.5 \%$ \\
& Emp & $14.3 \%$ & $16.9 \%$ & $8.3 \%$ & $9.1 \%$ & $12.7 \%$ & $22.3 \%$ \\
& GGO & $0.4 \%$ & $0.3 \%$ & $0.8 \%$ & $0.3 \%$ & $0.3 \%$ & $0.5 \%$ \\
& Fib & $2.0 \%$ & $1.7 \%$ & $2.0 \%$ & $1.4 \%$ & $2.2 \%$ & $2.7 \%$ \\
\hline
\end{tabular}




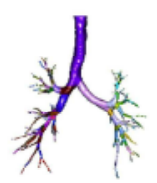

(a)

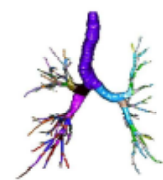

(b)

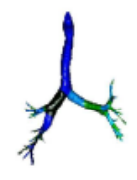

(c)

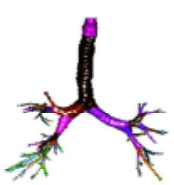

(d)

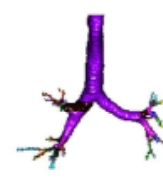

(e)

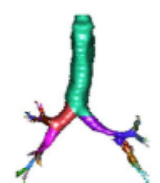

(f)

Figure 15: Extracted airway trees using the conventional 3D region growing algorithm. (a) NJ (Normal). (b) SK (UIP). (c) KS (COPD). (d) TK (IIPs). (e) SY (IIPs). (f) MJ (COPD).

Table 5: Airway extraction statistics for the results shown in Fig. 15. AV = airway volume $(c c), \mathbf{A L}=$ airway length $(\mathrm{mm}), \mathbf{N B}=$ number of bronchi

\begin{tabular}{|c|c|c|c|}
\hline Name & AV & AL & NB \\
\hline NJ & 50 & 2,109 & 287 \\
\hline SK & 63 & 2,601 & 372 \\
\hline KS & 37 & 807 & 127 \\
\hline TK & 54 & 1,563 & 216 \\
\hline SY & 46 & 796 & 167 \\
\hline MJ & 51 & 666 & 95 \\
\hline
\end{tabular}

identify that the method proposed identified a substantially larger airway volume than the conventional 3D region growing algorithm, with a $20.4 \%$ improvement on average. The method proposed detected a much longer airway tree, more than twice the airway tree length detected by the conventional 3D region growing algorithm. The number of bronchi detected by the method proposed was almost twice the result obtained from the conventional $3 \mathrm{D}$ region growing algorithm.

\subsubsection{Comparison with Commercial Softwares}

Figures 16.(a)-(f) show the results of the bronchus extraction algorithm using the commercial software AW 4.6 from GE Healthcare and Figs. 16.(g)(j) show similar results using the commercial software Bf-Navi from Olympus. The results determined by the Bf-Navi from Olympus and the method proposed had equivalent accuracy (see Figs. 10 and 16). The CT images from 


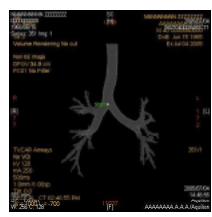

(a)

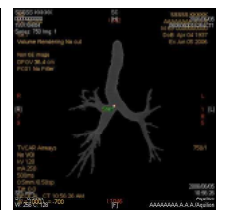

(b)

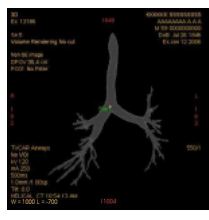

(c)

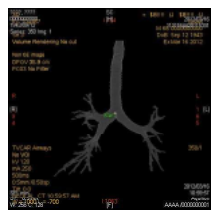

(d)

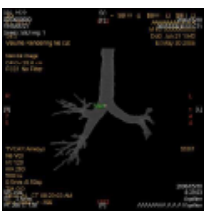

(e)

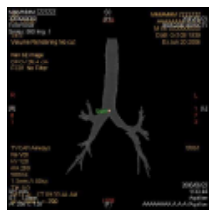

(f)

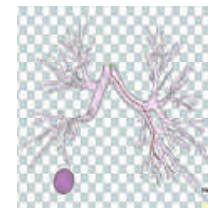

(g)

(h)

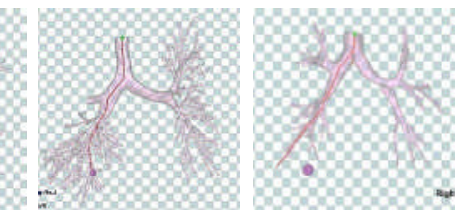

(i)

(j)

Figure 16: Extracted airway trees using other commercial software (a)-(f) AW 4.6 from GE Healthcare and (g)-(j) Bf-Navi from Olympus. (a)-(g) NJ (Normal). (b)-(h) SK (UIP). (c)-(i) KS (COPD). (d) TK (IIPs). (e) SY (IIPs). (f)-(j) MJ (COPD).

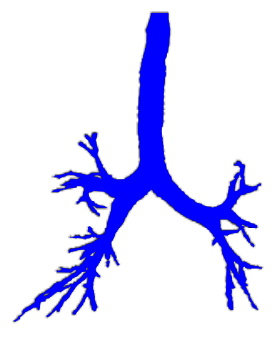

(a)

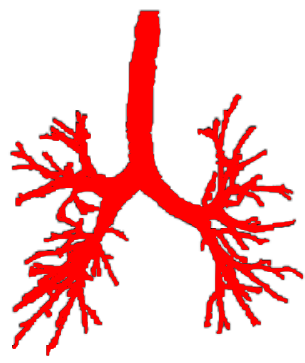

(b)

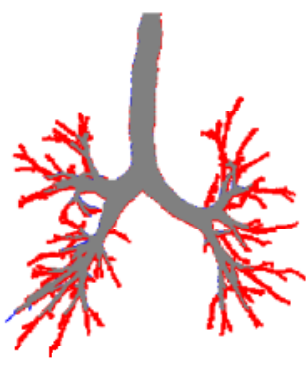

(c)

Figure 17: (a) Projected area of the airway determined by the AW 4.6. (b) Projected area of the airway determined by the proposed failure tracking and recovery algorithm. (c) Composition of both projected areas (a) and (b). 
Table 6: Airway extraction statistics (see Fig. 10). PA = projected airway area, FRA = proposed failure tracking and recovery algorithm projected airway area, AWA $=$ AW 4.6 projected airway area, FROA = projected airway area that is present exclusively in the method proposed, AWOA = projected airway area that is present exclusively in the AW 4.6 from GE Healthcare, $\mathbf{P T}=$ processing time $(s), \mathbf{F R}=$ proposed failure tracking and recovery method, $\mathbf{R G}=$ conventional $3 \mathrm{D}$ region growing algorithm, $\mathbf{B f}=$ Bf-Navi from Olympus and $\mathbf{A W}=\mathrm{AW} 4.6$ from GE Healthcare

\begin{tabular}{|c|c|c|c|c|c|c|c|}
\hline \multirow{2}{*}{ Name } & \multicolumn{4}{|c|}{ PA } & \multicolumn{3}{|c|}{ PT } \\
\cline { 2 - 9 } & FRA & AWA & FROA & AWOA & FR & Bf & AW \\
\hline NJ & $98.0 \%$ & $54.0 \%$ & $46.0 \%$ & $2.0 \%$ & 0.53 & 110 & 12 \\
\hline SK & $99.1 \%$ & $52.1 \%$ & $47.9 \%$ & $0.9 \%$ & 0.79 & 300 & 30 \\
\hline KS & $90.0 \%$ & $84.2 \%$ & $15.8 \%$ & $10.0 \%$ & 0.29 & 180 & 16 \\
\hline TK & $98.8 \%$ & $55.5 \%$ & $44.5 \%$ & $1.2 \%$ & 0.64 & & 29 \\
\hline SY & $96.4 \%$ & $79.3 \%$ & $20.7 \%$ & $3.6 \%$ & 1.09 & & 13 \\
\hline MJ & $96.2 \%$ & $93.3 \%$ & $6.7 \%$ & $3.8 \%$ & 0.25 & 60 & 16 \\
\hline
\end{tabular}

TK and SY were not processed by the Bf-Navi. Table 6 compares the processing time of the proposed method with the two commercial systems (AW 4.6 and Bf-Navi).

The proposed failure tracking and recovery algorithm determined the airway tree in less than one tenth of the runtime required by the AW 4.6 and less than one hundredth of the runtime required by the Bf-Navi. A comparison between the commercial systems is almost impossible because just the display output is available. A comparison between airway tree orthogonal views is used to compare the algorithm proposed and the AW 4.6 (the fastest tested commercial system). Initially, a coronal view of the extracted airway trees are created and the images are manually scaled such that both airways can be registered. Fig. 17.(a) shows the projected area of the airway determined by the AW 4.6, Fig. 17.(b) shows the projected area of the airway determined by the algorithm proposed and Fig. 17.(c) shows the composition of both projected areas. The common area is shown in gray. The area represented by the composition of both projected areas is represented as $100 \%$. Table 6 shows a summary of the determined projected area statistics. It is possible to observe that in all the cases the projected area determined by the algorithm proposed is at least $90 \%$ of the total area. Considering subjects NJ and SK, which have larger number of branches, the projected area obtained by the proposed algorithm showed to be twice as large. 


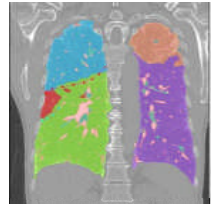

(a)

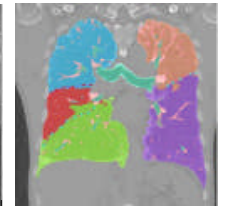

(b)

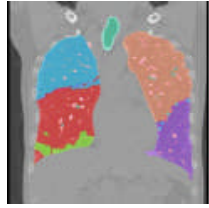

(c)

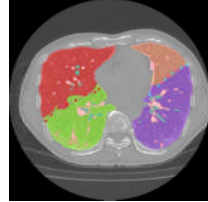

(d)

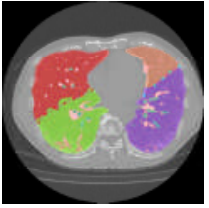

(e)

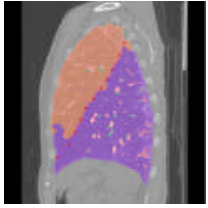

(f)

Figure 18: Classification of the lung into lobes for patient SK (UIP). (a)-(c) coronal images. (d)-(e) axial images. (f) sagittal image. A medical doctor draw a dotted line at the correct position for the inter-lobe fissures.

Table 7: Errors associated with the inter-lobe fissure determination comparing with a medical doctor evaluation. $\mathbf{R U L}=$ right upper lower fissure, $\mathbf{R U M}=$ right upper middle fissure, RML - right middle lower fissure, LUL - left upper lower fissure

\begin{tabular}{|c|c|c|c|c|}
\hline Name & RUL & RUM & RML & LUL \\
\hline NJ & $0.6 \%$ & $1.0 \%$ & $1.5 \%$ & $0.6 \%$ \\
\hline SY & & $2.0 \%$ & $2.5 \%$ & $9.0 \%$ \\
\hline KS & $2.2 \%$ & $2.2 \%$ & $5.9 \%$ & $0.7 \%$ \\
\hline TK & $2.1 \%$ & $2.5 \%$ & $2.9 \%$ & $6.5 \%$ \\
\hline SK & $0.8 \%$ & $1.0 \%$ & $0.9 \%$ & $0.6 \%$ \\
\hline MJ & $4.1 \%$ & $5.2 \%$ & $3.3 \%$ & $3.7 \%$ \\
\hline
\end{tabular}

\subsubsection{Manual Inter-lobe Fissure Determination}

Figs. 18.(a)-(f) show some 2D images with the lobe classification made by the system proposed. The same figures show the correct position of the interlobe fissures determined by a medical doctor in dotted lines for patient SK (UIP). The same procedure was executed for all CT data sets, and Table 7 shows the errors involved in the inter-lobes fissure determined automatically by the algorithm and defined by the medical doctor. In the case of SY (IIPs), the right upper lower fissure was very hard for the medical doctor to detect. The system proposed detected a surface with very small area, and this is why that space was left empty in Table 7 . The average error was $2.8 \%$.

\subsubsection{AW 4.6 Lobe Volume Comparison}

The AW 4.6 has a function which allows the user to determine the location of the lung fissures. Based on that information, AW 4.6 divides the lung into lobes. Lung and lobes volumes comparison between the result from the AW 
Table 8: Errors associated with the lung and lobe segmentation. The lung volume was compared with the result from AW 4.6 from GE Healthcare. The lobes were manually segmented by a medical doctor. $\mathbf{L V}=$ Lung Volume, $\mathbf{R U L}=$ right upper lobe, $\mathbf{R M L}=$ right middle lobe, $\mathbf{R L L}=$ right lower lobe, $\mathbf{L U L}=$ left upper lobe, $\mathbf{L L L}=$ left lower lobe

\begin{tabular}{|c|c|c|c|c|c|c|}
\hline Name & LV & RUL & RML & RLL & LUL & LLL \\
\hline NJ & $1.8 \%$ & $1.5 \%$ & $10.6 \%$ & $0.9 \%$ & $4.0 \%$ & $1.2 \%$ \\
\hline SY & $7.1 \%$ & $2.2 \%$ & $1.0 \%$ & $19.0 \%$ & $28.5 \%$ & $6.3 \%$ \\
\hline KS & $0.0 \%$ & $4.4 \%$ & $14.2 \%$ & $1.3 \%$ & $1.9 \%$ & $4.0 \%$ \\
\hline TK & $3.0 \%$ & $1.1 \%$ & $15.3 \%$ & $3.3 \%$ & $10.8 \%$ & $13.2 \%$ \\
\hline SK & $4.2 \%$ & $4.4 \%$ & $12.6 \%$ & $2.6 \%$ & $9.2 \%$ & $0.7 \%$ \\
\hline MJ & $0.3 \%$ & $1.9 \%$ & $0.3 \%$ & $2.1 \%$ & $1.0 \%$ & $3.0 \%$ \\
\hline
\end{tabular}

4.6 and from the system proposed are compiled in Table 8 . The average error among the lobe volumes is $6.1 \%$ and among the lung volumes, it is $2.7 \%$.

\subsubsection{Manual Lobe Injured Region Determination}

A medical doctor analyzed three slices from each of the processed 3D CT images and the amount of damage in the right lower lobe was determined. The medical doctor is determining the region associated with the right lower lobe and how much it is injured. The same slices were processed by the proposed system. The results were compiled in Table 9. The correlation coefficient between the injured amount determined in the right lower lobe by the medical doctor and by the system proposed was 0.941 . This shows strong correlation between both data sets.

\section{Conclusions and Future Works}

The algorithm proposed, based on failure tracking and recovering concept, was able to segment the airway trees from normal, IIPs, UIP and COPD subjects. Experiments demonstrate that the method proposed is able to segment airway trees more precisely than the conventional 3D region growing algorithm. Visually, the similar results are obtained with commercial systems. The processing time of the method proposed is much smaller when compared with the commercial systems. The same algorithm was used to segment the pulmonary vessels from the same subjects. The bronchial and vessel branches detected were classified into lobes. Using the Voronoi diagram, the lungs were separated into lobes. The determined inter-lobe fissures 
Table 9: Diffuse lung disease patterns percentages present in the right lower lobe. MD $=$ medical doctor, $\mathbf{P S}=$ proposed system, No = normal, GGO = ground-glass opacity, $\mathbf{E m p}=$ emphysema and $\mathbf{F i b}=$ fibrosis

\begin{tabular}{|c|c||c|c|c|c||c|c|c|c|}
\hline \multirow{2}{*}{ Name } & \multirow{2}{*}{ Slice } & \multicolumn{9}{|c||}{ MD } & \multicolumn{4}{c|}{ PS } \\
\cline { 3 - 9 } & & No & Emp & GGO & Fib & No & Emp & GGO & Fib \\
\hline \multirow{3}{*}{ SK } & 400 & $80 \%$ & $0 \%$ & $0 \%$ & $20 \%$ & $78.2 \%$ & $7.2 \%$ & $3.4 \%$ & $11.2 \%$ \\
& 310 & $90 \%$ & $0 \%$ & $0 \%$ & $10 \%$ & $85.8 \%$ & $4.5 \%$ & $4.6 \%$ & $5.1 \%$ \\
& 190 & $85 \%$ & $0 \%$ & $5 \%$ & $10 \%$ & $90.4 \%$ & $1.7 \%$ & $3.3 \%$ & $4.6 \%$ \\
\hline \multirow{3}{*}{ KS } & 165 & $95 \%$ & $5 \%$ & $0 \%$ & $0 \%$ & $84.6 \%$ & $2.4 \%$ & $8.2 \%$ & $4.8 \%$ \\
& 135 & $80 \%$ & $15 \%$ & $5 \%$ & $0 \%$ & $88.0 \%$ & $0.8 \%$ & $7.7 \%$ & $3.5 \%$ \\
& 90 & $85 \%$ & $10 \%$ & $5 \%$ & $0 \%$ & $92.5 \%$ & $0.6 \%$ & $3.4 \%$ & $3.5 \%$ \\
\hline \multirow{3}{*}{ TK } & 265 & $50 \%$ & $0 \%$ & $20 \%$ & $30 \%$ & $9.9 \%$ & $0.0 \%$ & $57.6 \%$ & $32.5 \%$ \\
& 205 & $50 \%$ & $0 \%$ & $20 \%$ & $30 \%$ & $1.6 \%$ & $0.0 \%$ & $76.4 \%$ & $22.0 \%$ \\
& 140 & $30 \%$ & $0 \%$ & $40 \%$ & $30 \%$ & $0.1 \%$ & $0.0 \%$ & $73.9 \%$ & $26.0 \%$ \\
\hline \multirow{3}{*}{ SY } & 355 & $90 \%$ & $0 \%$ & $0 \%$ & $10 \%$ & $87.2 \%$ & $1.6 \%$ & $6.9 \%$ & $4.3 \%$ \\
& 270 & $90 \%$ & $0 \%$ & $0 \%$ & $10 \%$ & $82.4 \%$ & $2.3 \%$ & $8.9 \%$ & $6.4 \%$ \\
& 180 & $85 \%$ & $0 \%$ & $0 \%$ & $15 \%$ & $78.0 \%$ & $2.9 \%$ & $12.2 \%$ & $6.9 \%$ \\
\hline \multirow{2}{*}{ MJ } & 180 & $100 \%$ & $0 \%$ & $0 \%$ & $0 \%$ & $97.5 \%$ & $2.4 \%$ & $0.0 \%$ & $0.1 \%$ \\
& 130 & $95 \%$ & $5 \%$ & $0 \%$ & $0 \%$ & $88.2 \%$ & $10.7 \%$ & $0.6 \%$ & $0.5 \%$ \\
& 75 & $100 \%$ & $0 \%$ & $0 \%$ & $0 \%$ & $83.4 \%$ & $12.9 \%$ & $1.0 \%$ & $2.7 \%$ \\
\hline
\end{tabular}


were compared with a medical doctor evaluation. The comparison showed very small error percentage. The algorithm proposed by Asakura et al. [13] was used to determine the presence of diffuse lung diseases. Finally, it was possible to determine how much of each lobe is injured. A final comparison of how much of the right lower lobe is injured between the proposed system and medical doctor evaluation, showed good correlation.

This preliminary work defined a research direction. Lung structures and injured regions were determined. The registration of the results with functional imaging is one current goal. It is a multi-modality registration in which it is necessary to overcome several problems: the breathing state can be different, the image quality is different with distinct properties.

\section{Acknowledgments}

We are indebted to all patients and all the residents working in our units during the study period for their dedication and collaboration in providing care to the patients that participated in this research. This project was partially supported by a joint project from JSPS/CAPES under the Japan - Brazil Research Cooperative Program and Grants-in-Aid for Scientific Research (24500539). The last author was partially supported by CNPq (309570/2010-7 and 471119/2010-5) and FAPESP (2010/19685-5).

\section{References}

[1] B. Raasch, E. Carsky, E. Lane, J. O'Callaghan, E. Heitzman, Radiographic anatomy of the interlobar fissure: a study of 100 specimens, American Journal of Roentgenology 138 (6) (1982) 1043-1049.

[2] R. Wiemker, T. Bülow, T. Blaffert, Unsupervised extraction of the pulmonary interlobar fissures from high resolution thoracic CT data, in: Proceedings of the $19^{\text {th }}$ International Congress Exhibition-Computer Assisted Radiology and Surgery, Berlin, 2005, pp. 1121-1126.

[3] J. Wang, M. Betke, J. P. Ko, Pulmonary fissure segmentation on CT, Medical Image Analysis 10 (4) (2006) 530-547.

[4] L. Zhang, E. A. Hoffman, J. M. Reinhardt, Atlas-driven lung lobe segmentation in volumetric X-ray CT images, IEEE Transactions on Medical Imaging 25 (1) (2006) 1-16. 
[5] E. M. van Rikxoort, B. van Ginneken, M. Klik, M. Prokop, Supervised enhancement filters: Application to fissure detection in chest CT scans, IEEE Transactions on Medical Imaging 27 (1) (2008) 1-10.

[6] J. M. Kuhnigk, H. K. Hahn, M. Hindennach, V. Dicken, S. Krass, H. O. Peitgen, Lung lobe segmentation by anatomy-guided 3D watershed transform, in: Proceedings of the SPIE Conference on Medical Imaging, San Diego, 2003, pp. 1482-1490.

[7] X. Zhou, T. Hayashi, T. Hara, H. Fujita, Automatic recognition of lung lobes and fissures from multislice CT images, in: Proceedings of the SPIE Conference on Medical Imaging, San Diego, 2004, pp. 1629-1633.

[8] T. Mitsa, J. Qian, Modeling 3D lung morphogenesis using fractal geometries and a boundary constraint, in: Proceedings of the $15^{\text {th }}$ Annual International Conference of the IEEE Engineering in Medicine and Biology Society, San Diego, 1993, pp. 568 -569.

[9] T. Schlathoelter, C. Lorenz, I. C. Carlsen, S. Renisch, T. Deschamps, Simultaneous segmentation and tree reconstruction of the airways for virtual bronchoscopy, in: Proceedings of the SPIE Conference on Medical Imaging: Image Processing, 2002, pp. 103-113.

[10] M. Sonka, W. Park, E. Hoffman, Rule-based detection of intrathoracic airway trees, IEEE T Med Imaging 15 (3) (1996) 314 -326.

[11] G. Agam, I. Armato, S.G., C. Wu, Vessel tree reconstruction in thoracic CT scans with application to nodule detection, IEEE T Med Imaging 24 (4) (2005) 486-499.

[12] C. Kirbas, F. K. H. Quek, A review of vessel extraction techniques and algorithms, ACM Computing Surveys 36 (2000) 81-121.

[13] A. Asakura, T. Gotoh, T. Iwasawa, K. Saito, H. Akasaka, Classification system of the CT images with non-specific interstitial pneumonia, Journal of the Institute of Image Electronics Engineers of Japan 33 (2004) $180-188$.

[14] T. Iwasawa, A. Asakura, F. Sakai, T. Kanauchi, T. Gotoh, Assessment of prognosis of patients with idiopathic pulmonary fibrosis by computeraided analysis of CT images, Journal of Thoracic Imaging 24 (2009) 216-222. 
[15] M. Schael, Invariant texture classification using group averaging with relational kernel functions, in: Proceedings of the $2^{\text {nd }}$ International Workshop on Texture Analysis and Synthesis, 2002, pp. 129-133.

[16] T. Lei, W. Sewchand, Statistical approach to X-ray CT imaging and its applications in image analysis. I. Statistical analysis of X-ray CT imaging, IEEE Transactions on Medical Imaging 11 (1) (1992) 53-61.

[17] T. Iwasawa, T. Ogura, F. Sakai, T. Kanauchi, T. Komagata, T. Baba, T. Gotoh, S. Morita, T. Yazawa, T. Inoue, CT analysis of the effect of pirfenidone in patients with idiopathic pulmonary fibrosis, European Journal of Radiologydoi:http://dx.doi.org/10.1016/j.ejrad.2012.02.014.

[18] T. Iwasawa, T. Ogura, H. Takahashi, A. Asakura, T. Gotoh, T. Yazawa, T. Inoue, Pneumothorax and idiopathic pulmonary fibrosis, Japanese Journal of Radiology 28 (9) (2010) 672-679.

[19] X. Wang, C. Fang, Y. Xia, D. Feng, Airway segmentation for lowcontrast CT images from combined PET/CT scanners based on airway modelling and seed prediction, Biomedical Signal Processing and Control 6 (1) (2011) 48-56.

[20] S. Hu, E. Hoffman, J. Reinhardt, Automatic lung segmentation for accurate quantitation of volumetric X-ray CT images, IEEE Transactions on Medical Imaging 20 (6) (2001) 490-498.

[21] S. Hu, E. A. Hoffman, J. M. Reinhardt, Automatic lung segmentation for accurate quantitation of volumetric X-ray CT images, IEEE Transactions on Medical Imaging 20 (2001) 490-498.

[22] C. L. Jackson, J. F. Huber, Correlated applied anatomy of the bronchial tree and lungs with a system of nomenclature, Chest 9 (4) (1943) 319326.

[23] H. Ledoux, Computing the 3D Voronoi diagram robustly: An easy explanation, in: Proceedings of the $4^{\text {th }}$ Annual International Symposium on Voronoi Diagrams in Science and Engineering, 2007, pp. $117-129$. 